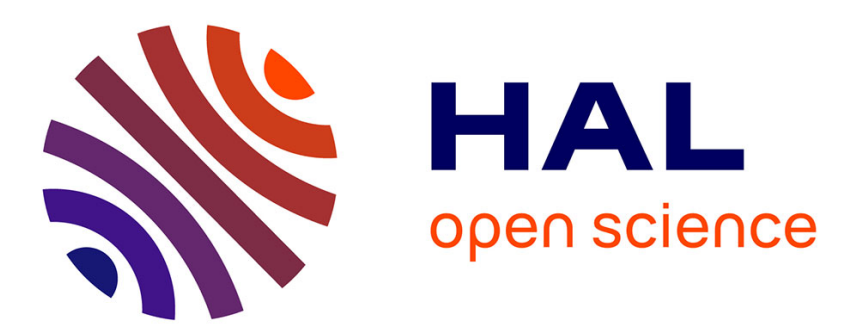

\title{
Harry Potter au Palais Royal? La lutte contre le terrorisme comme cape d'invisibilité de l'état d'urgence et la transformation de l'office du juge administratif
}

\author{
Stéphanie Hennette-Vauchez, Serge Slama
}

\section{- To cite this version:}

Stéphanie Hennette-Vauchez, Serge Slama. Harry Potter au Palais Royal? La lutte contre le terrorisme comme cape d'invisibilité de l'état d'urgence et la transformation de l'office du juge administratif. Les Cahiers de la justice, 2017, 2, pp.281-295. hal-01676051

\section{HAL Id: hal-01676051 \\ https://hal.parisnanterre.fr/hal-01676051}

Submitted on 5 Jan 2018

HAL is a multi-disciplinary open access archive for the deposit and dissemination of scientific research documents, whether they are published or not. The documents may come from teaching and research institutions in France or abroad, or from public or private research centers.
L'archive ouverte pluridisciplinaire HAL, est destinée au dépôt et à la diffusion de documents scientifiques de niveau recherche, publiés ou non, émanant des établissements d'enseignement et de recherche français ou étrangers, des laboratoires publics ou privés. 


\title{
Harry Potter au Palais Royal ? La lutte contre le terrorisme comme cape d'invisibilité de l'état d'urgence et la transformation de l'office du juge administratif
}

\author{
Stéphanie Hennette Vauchez \\ Serge Slama \\ Université Paris Nanterre \\ CREDOF (UMR 7074 Centre de théorie et analyse du droit)
}

Il fallait une contribution portant, dans cette livraison des Cahiers de la Justice, sur la justice administrative et le terrorisme. Si, naturellement, c'est en principe le juge judiciaire, et en premier lieu le juge pénal, qui est compétent en la matière, le terrorisme et les moyens de le combattre étant classiquement l'affaire de la loi pénale ${ }^{1}$, le juge administratif est néanmoins familier, de longue date, de contentieux ayant trait au terrorisme ${ }^{2}$; on dressait d'ailleurs il y a déjà plus de dix ans le constat d'un phénomène d' " administrativisation " de la lutte contre le terrorisme ${ }^{3}$. Mais par-delà ces éléments sinon classiques, dûment établis, on souhaite ici concentrer l'analyse sur les effets de la proclamation, le 14 novembre 2015, puis de l'installation dans la durée, de l'état d'urgence -dont on pose par hypothèse qu'ils ont singulièrement changé la donne. En effet, l'état d'urgence a, d'abord, été déclenché en réponse à des attaques terroristes d'ampleur. Les cinq lois de prorogation dont il a, ensuite, fait l'objet ont été l'occasion, et le projet de loi constitutionnelle de "protection de la Nation ", ont été justifiées, au plus haut niveau de l'Etat, par la permanence de la menace terroriste. Ces textes ont, tantôt, intégré dans le dispositif de la loi relative à l'état d'urgence de 1955, au nom de la lutte contre le terrorisme, des dispositifs déjà testés dans d'autres branches du droit (droit des étrangers, droit des fichiers...) ; c'est le cas des assignations à résidence ${ }^{4}$, accompagnées d'astreintes ou sous surveillance électronique ou encore, depuis la loi du 21 juillet 2016, des contrôles d'identité généralisés sur réquisition du préfet ${ }^{5}$ ou encore les interdictions de manifestation. Tantôt, les lois de prorogation de l'état d'urgence ont fourni un cadre à l'adoption de nouveaux dispositifs de lutte contre le terrorisme qui, débordant le cadre de la loi du 3 avril 1955, ont été insérés dans des dispositifs juridiques de droit commun (Code pénal, Code de procédure pénale, Code de la sécurité intérieure...) et sont donc désormais installés à titre permanent dans notre droit et continueront donc de produire des effets bien au-delà de l'état d'urgence. Les liens sont donc étroits entre état d'urgence et terrorisme... fussent-ils rhétoriques.

Car en effet, l'utilité réelle de l'état d'urgence dans la lutte contre le terrorisme est, de l'avis des spécialistes comme des instances d'évaluation et de contrôle, quasi-nulle. Le

\footnotetext{
${ }^{1}$ V. le dossier « Lutte contre le terrorisme », AJ pénal 2016. 407.

2 Pour une synthèse v. F. Nicoud, "Le terrorisme au filtre de la jurisprudence administrative », 2015 http://riseo.fr/IMG/pdf/2-2015/11-Nicoud-Le-terrorisme-devant-le-juge-administratif.pdf ; M. Sirinelli, « Police administrative et terrorisme : quel contrôle juridictionnel ? ", RFDA, 2013, p. 1024.

${ }^{3}$ F. Rolin, S. Slama, « Les libertés dans l'entonnoir de la législation anti-terroriste ", AJDA, 2006, p. 975.

${ }^{4}$ Article L 561-1 du CESEDA issu de la loi n²011-672 du 16 juin 2011 - art. 47.

${ }^{5}$ Depuis la loi du 21 juillet 2016, l'article 8-1 de la loi de 1955 habilite le préfet à autoriser des contrôles généralisés dans des zones déterminés, suivant le modèle des contrôles d'identité sur réquisition du Procureur de la République (art. 78-2 al. 6 du CPP).Voir à leur sujet le récent « recadrage » du Conseil constitutionnel : CC n²016-606/607 QPC, 24 janvier 2017, M. Ahmed M. et a. On sait que ces contrôles ont un fort potentiel discriminatoire.
} 
rapport statistique entre le nombre de mesures administratives prises en application de l'état d'urgence et le nombre de poursuites judiciaires effectivement déclenchées en matière terroriste est édifiant : la montagne a accouché d'une souris. L'ensemble des rapports et études produits depuis dix-huit mois, qu'ils émanent de la société civile ${ }^{6}$ ou des institutions publiques ${ }^{7}$, converge pour établir que, passés les premiers jours suivant le déclenchement de l'état d'urgence, les mesures que la mise en application de la loi du 3 avril 1955 a permis de prendre n'ont pas ciblé spécifiquement les réseaux terroristes. Bien plutôt, celles-ci ont été dirigées vers des objectifs en lien avec la surveillance, la discipline et la mise au pas de divers groupes perçus comme menaçant, de manière générale, l'ordre public : cellules de religieux radicalisés, cellules d'activistes politiques (notamment dans la mouvance anarcho-libertaire avec le prétexte de la COP 21, ou pour canaliser le mouvement social : mouvements d'opposition au projet d'aéroport à Notre Dame des Landes, à la loi El Khomri, Nuit Debout ${ }^{8}$ ), fauteurs de troubles en tous genres (supporters de foot, militants de la cause des sans-papiers...). L'état d'urgence a ainsi rapidement et désormais durablement permis des coups de pied dans diverses fourmilières. Bien plus qu'il ne se révèle comme outil de la lutte contre le terrorisme ${ }^{9}$, il a constitué une technique de police, et se différencie peu, en cela, des modes d'intervention observés dans l'affaire Tarnac ou s'agissant de l'opposition au barrage de Sivens.

Ces précisions liminaires sont essentielles au propos que l'on souhaite développer ici, et visent à dégoupiller le piège des mots : on s'intéresse ici à ce que l'état d'urgence, en tant qu'il est présenté, prorogé, légitimé (....) par la lutte contre le terrorisme, fait à la justice administrative. En d'autres termes, si l'on propose ici une contribution au numéro des Cahiers de la justice consacré à la lutte contre le terrorisme, c'est en pleine conscience de ce que ce paradigme rhétorique fait office de cape d'invisibilité dissimulant l'état d'urgence. La chose mérite d'être tentée, car il ne fait guère de doute que ce curieux attelage d'un régime d'exception instrumentalisé par (ou instrumentalisant ?) l'aspiration du peuple à la sécurité, s'il ne bouleverse pas tout ce que l'on sait de la justice administrative et de ses modes de fonctionnement (I), altère tout de même singulièrement l'office du juge (II) qui peine à cantonner les effets de dissémination d'une logique de l'exception au cœur de l'appareil administratif (III).

\section{Le recours aux procédés classiques de contrôle des mesures de police en période d'exception}

Selon les rapporteurs du contrôle parlementaire de l'état d'urgence, "le contentieux dévolu au juge administratif [dans le cadre de l'état d'urgence] [était] entièrement

\footnotetext{
${ }^{6}$ V. notamment l'important : Amnesty international, Des vies bouleversées: L'impact disproportionné de l'état d'urgence en France, Rapport, 4 février 2016.

${ }^{7}$ V. notamment la $2^{\text {ème }}$ communication d'étape sur le contrôle de l'état d'urgence (Commission des Lois, 13 janvier 2016, J.-J. Urvoas : http://www2.assembleenationale.fr/static/14/lois/communication 201601 13.pdf), puis les deux rapports sur le contrôle parlementaire de l'état d'urgence (D. Raimbourg, J.-F. Poisson, 25 mai 2016 et 6 décembre 2016).

${ }^{8} \mathrm{~V}$. la dizaine d'arrêtés du préfet de Police d'interdiction fondés sur la loi de 1955 https://www.prefecturedepolice.interieur.gouv.fr/Nous-connaitre/Salle-de-presse/Arretes/Liste-des-arretes

${ }^{9}$ Certains auteurs considèrent d'ailleurs que non seulement l'état d'urgence ne sert pas la lutte contre le terrorisme mais qu'elle l'affaiblit : V. Sizaire, "Quand parler de " terrorisme » ? ", Le Monde diplomatique, août 2016.
} 
nouveau ${ }^{10}$. Pourtant à y regarder de près, confronté très rapidement à un flux de requêtes émanant des personnes assignées à résidence, interdites de séjour ou perquisitionnées, le premier réflexe du juge administratif a été de recourir aux procédés classiques de contrôle des mesures de police administrative en période d'exception. Dès lors que sa compétence était acquise - elle a même été consolidée- (A.), la juridiction administrative a effectué le contrôle de ces mesures, dans un cadre rapidement clarifié par le Conseil d'Etat, celui du contrôle classique proportionnalité (C.), dans le cadre privilégié du référé-liberté (B).

\section{A. La consolidation de la compétence du juge administratif sur l'ensemble des mesures prises dans le cadre de l'état d'urgence}

C'est d'emblée que le juge administratif s'est reconnu compétent pour connaître de la légalité des mesures adoptées sur le fondement de la loi du 3 avril 1955'11. A l'occasion de l'état d'urgence de 2005, il avait justifié son contrôle en soulignant que même si l'état d'urgence est un "régime de pouvoirs exceptionnels ", ses modalités de mise en oeuvre ne peuvent échapper dans un Etat de droit à tout contrôle de la part du juge administratif ${ }^{12}$.A l'occasion de l'actuel état d'urgence, la compétence du juge administratif est désormais consolidée par la loi : la loi du 20 novembre $2015^{13}$ pose que "l'ensemble des mesures prises sur le fondement du régime législatif de l'état d'urgence sont soumises au contrôle du juge administratif dans les conditions fixées par le code de justice administrative, notamment son livre $V$ [référés] $»^{14}$.

En tout état de cause l'ensemble des interprétations jurisprudentielles allaient de toute façon en ce sens -y compris au regard des -nombreuses- mesures prises entre le déclenchement de l'état d'urgence (14 novembre) et l'entrée en vigueur de cette première loi de prorogation (21 novembre). Cette compétence du juge administratif ne s'imposait pourtant pas avec la force de l'évidence, compte tenu du renforcement, dès la loi du 20 novembre 2015, des obligations liées à l'assignation à résidence (douze heures par jour à domicile, trois passages au commissariat par jour, obligation de demeurer dans le ressort de la commune sauf autorisation expresse du préfet) ou l'impact sur la vie privée et familiale et l'activité professionnelle des perquisitions administratives (cf. notamment la collecte des données à caractère personnel). Pourtant, dans le prolongement de la jurisprudence du Conseil $d^{\prime} E t a t^{15}$, le Conseil constitutionnel a estimé que ces mesures relèvent " de la seule police administrative » et ne peuvent avoir « d'autre but que de préserver l'ordre public et de

\footnotetext{
${ }^{10}$ Ass. nat., Contrôle parlementaire de l'état d'urgence, Rapport d'information n4281, par D. Raimbourg, J-F. Poisson, 6 déc. 2016, p. 99.

${ }^{11}$ CE, Ass., 16 déc. 1955, Bourokba.

${ }^{12} \mathrm{CE}$, ord., 14 nov. 2005, Rolin, n²86835.

${ }^{13}$ Loi n ${ }^{\circ}$ 2015-1501 du 20 novembre 2015 prorogeant l'application de la loi n 55-385 du 3 avril 1955 relative à l'état d'urgence et renforçant l'efficacité de ses dispositions.

${ }^{14}$ Loi du 3 avril 1955, art. 14-1. Cette compétence trouve bien sûr une limite puisque le juge administratif ne saurait connaître des sanctions pénales prononcées sur le fondement de l'article 13 de la loi de 1955 en cas de non-respect des mesures prescrites dans le cadre de l'état d'urgence.

${ }^{15}$ CE, ord., 11 déc. 2015, C. Domenjoud, $\mathrm{n}^{\circ}$ 395009. La compétence du juge administratif est apparue tellement évidente que lorsque le gouvernement avait souhaité inscrire à l'article 36-1 de la Constitution que ces décisions sont placées " sous le contrôle du juge administratif », le Conseil d’Etat a estimé cette précision " inutile " s'agissant de mesures de police administrative " placées naturellement sous le contrôle du juge administratif » (Avis sur le projet de loi constitutionnelle de protection de la Nation du 11 décembre 2015, cons. 11).
} 
prévenir les infractions ${ }^{16}$. Il juge, de manière contestable, qu'il ne s'agit pas de mesures " privatives " de la liberté individuelle au sens de l'article 66 de la Constitution ${ }^{17}$ et peuvent, dès lors, relever de la compétence exclusive du juge administratif. Vis-à-vis des autres mesures prévues par la loi de 1955, sa position est à l'avenant : ni les perquisitions administratives ${ }^{18}$, ni les décisions préfectorales de fermeture provisoire des salles de spectacles, débits de boissons et lieux de réunion ${ }^{19} \mathrm{ni}$, certainement, les interdictions de séjour ${ }^{20}$ ne portent atteinte à la liberté individuelle.

\section{B. L'utilisation privilégiée du référé-liberté}

En voie de consolidation permanente depuis sa consécration en 2000, la procédure de référé-liberté s'est, sans surprise, retrouvée " en première ligne ${ }^{21}$ du contentieux de l'état $d^{\prime}$ urgence ${ }^{22}$. Il est rapidement devenu l'instrument privilégié des assignés ou des interdits de séjour pour contester les mesures pesant sur eux. Ce n'est pas pour autant allé sans difficultés; notamment, dans un premier temps, on a vu les juges des référés-liberté rejeter les premières requêtes au " tri " pour " défaut d'urgence " ${ }^{23}$. Le Conseil d'Etat réagit toutefois rapidement : dès le 11 décembre, et après de sévères critiques du rapporteur public ${ }^{24}$, les ordonnances concernant les assignés de la COP 21 furent toutes censurées. Même, la section du contentieux a posé en la matière, à rebours l'évolution jurisprudentielle actuelle, une présomption d'urgence et effectué un contrôle de proportionnalité (mais rejeté ensuite, au fond, les sept requêtes). Ces censures, complétées par la circulaire adressée par le Conseil d'Etat aux juridictions inférieures afin qu'elles se préparent à traiter rapidement du contentieux de l'état d'urgence avec des magistrats expérimentés ${ }^{25}$, ont constitué des signaux suffisamment forts pour que les juges des référés de première instance se décident à saisir le cognard en vol et à exercer un réel contrôle sur les mesures contestées devant eux.

Il importe toute de même de préciser que cette voie privilégiée du référé-liberté perd considérablement de son utilité vis-à-vis de la mesure la plus utilisée sous l'état d'urgence : les perquisitions administratives. En effet en vertu du privilège du préalable, la perquisition a déjà eu lieu lorsque le juge des référés peut être matériellement saisi. Si le Conseil d'Etat a précisé de manière constructive précisé le régime d'indemnisation des préjudices éventuellement causés par une perquisition ${ }^{26}$, il reste qu'on ne saurait identifier, en la matière, de recours effectif -et ce, quoiqu'en dise le Conseil ${ }^{27}$. Seul un régime d'autorisation

\footnotetext{
${ }^{16}$ Cons. constit., déc. $n^{\circ}$ 2015-527 QPC du 22 décembre 2015, M. Cédric D. , cons. 5.

${ }^{17} \mathrm{Ibid}$. cons. 5 et 6 . Le Conseil constitutionnel précise toutefois qu'une loi prévoyait que l'intéressé serait astreint à rester à domicile plus de douze heures par jour, cela constituerait une privation de la liberté individuelle nécessitant l'intervention du juge judiciaire en vertu de l'article 66 de la Constitution.

${ }^{18}$ Cons. constit., déc. $\mathrm{n}^{\circ} 2016-536$ QPC du 19 février 2016, LDH, cons. 4 ;

${ }^{19}$ Cons. constit., déc. $n^{\circ}$ 2016-535 QPC du 19 février 2016, LDH, cons. 6.

${ }^{20}$ Plusieurs QPC ont été renvoyées par des tribunaux administratifs au Conseil d'Etat portant sur l'article 5 et I'article 13 (sanction pénale) de la loi de 1955 (TA Rennes, ord., 17 janv. 2017, n¹604924QPC ; TA Montreuil, ord., 18 janv. 2017, n¹606585).

${ }^{21}$ X. Domino, concl. sur CE, Sect., 11 déc. 2015,. Domenjoud, RFDA, 2016 p. 105

${ }^{22}$ En 2005, le référé-liberté avait été utilisé, sans succès, pour obtenir la fin anticipée de l'état d'urgence : CE, réf., 9 déc. 2005, Allouache et a., $n^{\circ} 287777$.

${ }^{23}$ Comme on a pu le voir dans 6 des 7 dossiers des assignés de la COP21.

${ }^{24} \mathrm{~V}$. Concl. X. Domino, préc..

${ }^{25}$ Circulaire évoquée par Jean-Marc Sauvé lors de son audition à l'Assemblée nationale le 8 janvier 2016 Rapport $n^{\circ} 3784$.

${ }^{26}$ CE, Ass, 6 juillet 2016, Napol et ., $\mathrm{n}^{\circ} 398234$.

${ }^{27}$ Cons. constit., déc. $n^{\circ}$ 2016-536 QPC, préc.., cons. 11.
} 
préalable par un juge, de préférence judiciaire mais possiblement aussi administratif comme c'est désormais le cas pour les assignations de plus d'un an (v. infra), serait susceptible d'assurer un recours réellement effectif.

\section{Le triple test de proportionnalité (une façade ?)}

Habitué à restreindre l'intensité de son contrôle dans le cadre des régimes d'exception ${ }^{28}$, le juge administratif n'exerçait jusque-là sur les mesures prises dans le cadre de l'état d'urgence qu'un contrôle restreint, dit de l'erreur manifeste d'appréciation ${ }^{29}$. Mais depuis les décisions rendues le 11 décembre 2015, le juge administratif exerce désormais le " triple test " de proportionnalité aussi bien pour les assignations à résidence ${ }^{30}$ que pour les perquisitions $^{31}$ : ces mesures doivent être nécessaires, adaptées et proportionnées. Le contrôle de proportionnalité est pris en compte par le juge constitutionnel pour estimer que ces dispositions sont conformes aux droits et libertés que la Constitution garantit ${ }^{32}$.

Le juge administratif devrait aussi vérifier, s'il était fidèle à l'arrêt Benjamin ${ }^{33}$, que des mesures moins attentatoires aux libertés ne pourraient être prises ${ }^{34}$; mais cette part-là de sa jurisprudence semble mise de côté sous l'état d'urgence puisqu'au contraire, il a accepté dans l'arrêt Domenjoud que joue alors la « logique opérationnelle » (v. infra).

Cela étant, à bien y regarder, la consécration de ce triple test de proportionnalité ne change pas fondamentalement la donne et aboutit rarement à ces censures. Certes, selon le bilan statistique officiel au 4 janvier $2017^{35}$, le juge des référés du Conseil d'Etat a donné gain de cause aux assignés dans 9 des 60 recours introduits devant lui (dont 4 annulations ou suspensions partielles ${ }^{36}$ ). Dans sa lecture des statistiques, Jean-Marc Sauvé ajoute à ces annulations ou suspensions les 17 non-lieux à statuer, pour en conclure qu' « en appel devant le Conseil d'État, le taux de remise en cause des assignations s'est élevé à $56 \% "{ }^{37}$.

Mais en réalité, le bilan n'est pas aussi glorieux que celui dressé par le Severus Rogue du Conseil d'Etat ${ }^{38}$. Le nombre réel de suspensions par le juge des référés en appel est faible (5).

\footnotetext{
${ }^{28}$ CE, 28 juin 1918, Heyriès, nº 63412 ; CE 28 févr. 1919, Dames Dol et Laurent, nº 61593.

${ }^{29} \mathrm{CE}, 25$ juill. 1985, Mme Dagostini, $\mathrm{n}^{\circ}$ 68151. Ce contrôle de l'erreur manifeste a été maintenu aussi bien pour la décision de président de la République de promulgation de l'état d'urgence que pour celle de ne pas I'interrompre (CE, ord., 9 déc. 2005, Mme Allouache, préc.; CE, 27 janv. 2016, LDH, n 396220).

${ }^{30}$ CE, Sect., 11 déc. 2015, C. Domenjoud, préc., cons. 27.

${ }^{31} \mathrm{CE}$, avis du 6 juillet 2016, préc., cons. 6.

${ }^{32}$ Cons. constit., déc. $\mathrm{n}^{\circ}$ 2015-527 QPC, préc., cons. 12 ; déc. $\mathrm{n}^{\circ}$ 2016-536 QPC, préc., cons. 10.

${ }^{33} \mathrm{CE}, 19$ mai 1933, Benjamin et syndicat d'initiative de Nevers, $\mathrm{n}^{\circ} 17413$.

${ }^{34}$ Comme cela est rappelé dans l'intervention de J.-M. Sauvé, préc., p.5.

${ }^{35}$ Contentieux des mesures prises sur le fondement de la loi du 3 avril 1955, Données au 4 janvier 2017 reproduit dans $\mathrm{CNCDH}$, Avis sur le suivi de l'état d'urgence et les mesures anti-terroristes de la loi du 21 juillet 2016, p. 9.

${ }^{36} \mathrm{~V}$. pour un exemple de suspension partielle des modalités d'une assignation portant une atteinte grave et manifestement illégale au droit au respect de sa vie familiale ainsi qu'à l'intérêt supérieur de ses enfant (CE, réf., 6 janv. 2016, $\mathrm{n}^{\circ}$ 395622).

${ }^{37}$ Ceux-ci ne sont toutefois pas systématiquement favorables aux assignés. En effet un référé-liberté aboutit à un non lieu si l'administration a procédé à l'abrogation de l'assignation avant l'audience (souvent pour éviter la suspension en raison d'une illégalité). Mais la plupart du temps le premier arrêté abrogé est remplacé par un nouvel arrêté purgé de son illégalité (par exemple concernant le nombre de passages au commissariat ou en raison d'un problème de compétence du signataire de l'acte) sans que l'intéressé ne soit concrètement jamais sorti du régime d'assignation.

${ }^{38}$ Dans le cadre d'une recherche menée actuellement par le CREDOF pour le Défenseur des droits (" Ce qui reste(ra) toujours de l'urgence "), nous analysons les centaines de décisions rendues par les tribunaux
} 
Significativement, aucun des assignés de la COP 21 n'a obtenu satisfaction Mais surtout ce n'est pas en exerçant le triple test que le juge des référés aboutit à ces suspensions, mais en se plaçant sur le terrain de la matérialité des faits. Un nombre significatif de suspensions est en effet fondé sur le fait que les « notes blanches » sur lesquels l'administration s'est fondée pour ordonner la mesure en cause sont parfois insuffisamment précises et circonstanciées 39 . La valeur probante de ces notes blanches, et le poids qu'elles prennent dans la majeure partie des dossiers contentieux liés à l'état d'urgence, constitue d'ailleurs l'un des éléments les plus saillants de l'exceptionnalisation du contentieux administratif associée à l'activité des services de renseignement vis-à-vis des réseaux « terroristes ».

\section{L'exceptionnalisation de l'office du juge administratif de l'état d'urgence}

L'exceptionnalisation de l'office du juge administratif dans le cadre du contentieux des mesures prises dans le cadre de l'état d'urgence se donne à voir sous deux angles. Le premier est celui de l'accentuation de procédés déjà existants qui, souvent, ont déjà été éprouvés en droit des étrangers ou dans le contentieux des fichiers (A.). Le second est celui de la consécration de nouveaux rôles du juge administratif, qui signent le retour à un rôle archaïque de juge-administrateur et court-circuitent l'intervention a priori du juge judiciaire (B.).

\section{A. Accentuation de techniques de contrôles existantes}

Le premier élément saillant du contrôle opéré par le juge administratif sur les mesures prises dans le cadre de l'état d'urgence est le traitement qu'il réserve au recours quasisystématique, par l'administration, à des informations émanant de services de renseignement et qui prennent la forme de "notes blanches ", c'est-à-dire des notes policières non signées et non sourcées ou, alternativement et notamment dans les cas de radicalisation en prison, $d^{\prime}$ informations soumises par les services pénitentiaires ${ }^{40}$. Alors même que l'usage de ces notes policières est particulièrement critiqué et a même été proscrit par une circulaire de $2004^{41}$, le Conseil d'Etat leur reconnaît une valeur probante. Il pose comme seules conditions que le contenu de la note soit soumis au contradictoire, et que ne soient considérées comme probants par le juge administration les seuls faits " précis et circonstanciés " qui ne sont pas " sérieusement contestés par le requérant", à l'exclusion de toute interprétation ou extrapolation de la part des services. L'utilisation de ces notes blanches est de longue date ${ }^{42}$ courante dans le contentieux de l'expulsion des étrangers, et particulièrement des

\footnotetext{
administratives et cours administratives d'appel dans le cadre de l'état d'urgence proclamé depuis le 14 novembre 2015.

${ }^{39} \mathrm{~V}$. par ex. I'affaire particulièrement médiatisée (utilisation d'un portable aux abords du domicile d'une personnalité de Charlie hebdo) : CE, réf., 22 janvier 2016, $n^{\circ} 396116$. Voir pour les autres suspensions sur I'inexactitude matérielle des faits : CE, réf. 15 avril 2016, n³98377; CE, réf., 20 mai 2016, n 399692.

${ }^{40}$ Sur l'intégration de l'administration pénitentiaire à la famille du renseignement cf. M. Quinquis, "Le plan 'Urvoas' : un pas de plus dans la transformation policière de l'administration pénitentiaire ", La Revue des droits de l'homme [En ligne], Actualités Droits-Libertés, 10 janv. 2016. URL : http://revdh.revues.org/2862.

${ }^{41}$ Concl. X. Domino, préc.

${ }^{42}$ CE, ass., 11 oct. 1991, Ministre de l'intérieur c/ Diouri, n 128128 ; RFDA 1991. 978, concl. M. de Saint Pulgent.
} 
" terroristes » ou imams étrangers ${ }^{43}$. II n'est dès lors pas étonnant de voir la section du Conseil d'Etat confirmer cette jurisprudence dans l'arrêt Domenjoud et l'appliquer aux mesures de l'état d'urgence ${ }^{44}$. Il n'est pas davantage surprenant que le Conseil Constitutionnelle abonde ${ }^{45}$. Reste que l'état d'urgence de 2015/2017 a fait passer ce procédé d'un stade artisanal (quelques arrêtés ministériels d'expulsion) à l'ère industrielle : les notes blanches sont quasi systématiquement utilisées dans les arrêtés d'assignation à résidence, d'interdiction de séjour ou de perquisitions. Et sauf dans les rares cas où le requérant, souvent grâce à des avocats tenaces capables de mobiliser les techniques des pénalistes pour " démonter " la version policière, le juge administratif fait généralement siens les éléments factuels rapportés par les notes blanches.

Le deuxième élément saillant est l'acceptation par le Conseil d'Etat, de la logique " opérationnelle », proposée par le rapporteur public Xavier Domino ${ }^{46}$ et qui était déjà sousjacente à l'ordonnance Allouache du 9 décembre 200547. Dans les arrêts du 11 décembre 2015, le juge administratif suprême accepte que la liberté d'aller et venir des assignés à résidence soit restreinte non pas parce que leur comportement représente un trouble d'une ampleur telle qu'il ne pourrait être canalisé par les procédés de police ordinaire, mais parce que, dans le contexte de l'état d'urgence et de l'organisation de la COP 21, les forces de l'ordre sont « particulièrement mobilisées pour lutter contre la menace terroriste et parer au péril imminent ayant conduit à la déclaration de l'état d'urgence ${ }^{48}$. L'admission de cette logique est particulièrement liberticide - puisqu'elle permet dans l'absolu aux autorités de police d'interdire à peu près tout et n'importe quoi dès lors qu'il suffit d'invoquer l'occupation des forces de l'ordre à d'autres tâches ${ }^{49}$. Elle constitue aussi à proprement parler l'anti-arrêt Benjamin, puisque la restriction de police devient la règle au détriment de l'exercice des libertés. Consacrée par le juge administratif, cette logique "opérationnelle " a, depuis, reçu l'onction législative pour ce qui est des manifestations sur la voie publique : depuis la loi du 21 juillet 2016, on lit à l'article 8 de la loi du 3 avril 1955 que : "Les cortèges, défilés et rassemblements de personnes sur la voie publique peuvent être interdits dès lors que l'autorité administrative justifie ne pas être en mesure d'en assurer la sécurité compte tenu des moyens dont elle dispose ".

Le troisième élément remarquable est le développement d'une logique déjà décrite à l'occasion des affaires dites Dieudonné ${ }^{50}$ ou, préalablement, de la "Soupe aux cochons ${ }^{51}$ : la transformation de la police administrative d'un instrument préventif ou curatif des troubles à l'ordre public réel ou immatériel (morale, dignité) à un instrument prédictif du

\footnotetext{
${ }^{43}$ CE, 3 mars 2003, Ministre de l'intérieur c/ Rakhimov, n²38662 ; v. aussi O. Lecucq, " Les notes des renseignements généraux peuvent fonder une décision d'expulsion », AJDA 2005, p. 98.

${ }^{44}$ CE, Ass., 11 déc. 2015, Domenjoud, préc.

${ }^{45}$ Cons. constit., déc. $\mathrm{n}^{\circ}$ 2015-527 QPC, préc., cons. 15.

${ }^{46} \mathrm{X}$. Domino, concl. préc.

${ }^{47} \mathrm{CE}$, réf., 9 décembre 2005, Allouache et a., préc.

${ }^{48}$ CE, Sect., 11 déc. 2005, Domenjoud, préc., cons. 29.

${ }^{49}$ Ainsi, un maire a interdit, dans le contexte de l'état d'urgence, le survol du stade et de l'hôtel thalasso occupé par l'Equipe de football d'Espagne durant l'Euro par les drones et les appareils d'aéromodélisme (TA Poitiers, 9 nov. 2016, $n^{\circ} 1601342$ ).

${ }^{50}$ CE, réf., 9 janv. 2014, Ministre de l'intérieur c/Les Productions de la Plume et M. D. M'Bala M'Bala, préc. ; CE, 9 nov. 2015, Association générale contre le racisme et pour le respect de l'identité française chrétienne (AGRIF) et a., $\mathrm{n}^{\circ} 376107$; AJDA 2015. 2508, concl. A. Bretonneau.

${ }^{51} \mathrm{CE}$, ord., 5 janv. 2007, Ministre de l'intérieur c/ Association « Solidarité des Français », n 300311.
} 
risque virtuel, ce qu'on pourrait appeler, par facilité de langage, la logique Minority report ${ }^{52}$. Dans l'affaire Dieudonné, la mesure de police administrative était fondée sur le risque allégué qu'une infraction risquait d'être commise à l'occasion du spectacle donné par l'intéressé, compte tenu du fait qu'il était coutumier du fait et avait déjà été condamné pour cette raison. Dans le contentieux de l'état d'urgence, on voit le juge administratif confirmer la légalité d'arrêtés d'assignation à résidence ou d'ordres de perquisition en référence au profil et à la personnalité des requérants et à leurs fréquentations de certains milieux radicalisés tels qu'ils sont dépeints par l'administration à partir des informations des services de renseignement. Ainsi, le juge va typiquement considérer qu'eu égard au contexte de l'état d'urgence, il « existait de sérieuses raisons de penser que [son] comportement constitue une menace grave pour la sécurité et l'ordre publics ». En d'autres termes, il apparaît que, dès lors que le juge administratif tient pour suffisamment établis les faits reprochés, les éléments éventuellement invoqués par le requérant tendant à montrer qu'il s'est amendé ou à évolué ou qu'il n'épouse pas réellement ou totalement la cause radicale pèsent de peu de poids dans la balance face aux indices de "dangerosité potentielle ». Tout au plus obtient-il, dans certains cas et notamment lorsqu'il y a des justifications professionnelles, un aménagement de la mesure d'assignation à résidence (réduction du nombre de pointages quotidiens au commissariat, changement des heures de pointage pour les adapter à la situation professionnelle ou aux études suivies...).

Il faut enfin encore mentionner une dernière spécificité du contentieux de l'état d'urgence qu'est l'anonymisation des magistrats administratifs ayant rendu la décision, y compris parfois sur les décisions accessibles uniquement sur Ariane archive ${ }^{53}$, qui n'est normalement consultable que par les personnels des juridictions administratives ${ }^{54}$. On constate aussi que ce contentieux est fréquemment jugé par des magistrats particulièrement expérimentés - présidents de chambre ou chef de juridiction, ou en formation de trois juges des référés. Parallèlement, lorsqu'ils sont jugés au fond, les dossiers sont souvent rapportés par le président de la formation de jugement lui-même.

\section{B. Nouvelles formes d'intervention}

Mais il n'y a pas que les techniques de contrôle contentieux qui illustrent la transformation de l'office du juge ; de manière bien plus structurelle, ce sont les contours même de cet office qui ont subi, à la faveur de l'intensification récente de la lutte contre le terrorisme, de substantielles redéfinitions... lesquelles semblent converger dans le sens d'une accentuation des fonctions d'administrateur du juge administratif.

Déjà, la loi « renseignement » du 24 janvier 2015 avait charrié son lot de bouleversements : par-delà les questions de fond (définition et finalités des techniques de renseignement), elle a créé une formation spécialisée, habilitée secret défense, au Conseil d'Etat, compétente pour connaître des recours formés contre les décisions de la Commission

\footnotetext{
52 Dans la nouvelle de Ph. K. Dick, et le film de S. Spielberg, l’organisation gouvernementale Précrim repose sur la faculté des précogs (précognition) de connaître l'avenir et non, à proprement parler, de prédire un crime.

${ }^{53}$ Cette base de jurisprudence donne accès à la quasi-totalité des décisions rendues par les juridictions administratives (TA, CAA) dans des versions sans anonymisation du nom des parties.

${ }^{54} \mathrm{~V}$. dans le même sens les nouveaux articles L. 212-1 du code des relations entre le public et l'administration et L.773-9 du Code de la justice administrative qui permettent, depuis la loi n²017-258 du 28 février 2017 relative à la sécurité publique, d'anonymiser les décisions administratives fondées sur des motifs en lien avec la prévention d'actes de terrorisme y compris dans le cadre du contentieux administratif.
} 
nationale de contrôle des techniques de renseignement $(\text { CNCTR })^{55}$. Certes, la fonction de cette formation du Conseil d'Etat demeure contentieuse ; cela étant, les spécificités du contrôle doivent être soulignées qui annoncent, si ce n'est expriment, un rapprochement net entre fonction de juger et fonction d'administrer... Qu'on en juge : si « toute personne ayant un intérêt personnel et direct " peut en effet demander à savoir si des techniques de surveillance sont mises en œuvre à son endroit (art. 833-3 CSI), les réponses qu'elle recevra seront totalement opaques. Les dix premiers arrêts rendus, le 19 octobre 2016, par cette formation spéciale le confirment : les requérants qui cherchaient à faire établir le caractère illégal de la surveillance dont ils faisaient l'objet se sont vus laconiquement répondre que : " il a été procédé à la vérification demandée par... " (v. par ex. CE, 19 oct. 2016, n³96958). Impossible, dès lors, pour le requérant de réellement savoir s'il a fait l'objet d'une mesure de surveillance ou si, le cas échéant, la surveillance était bien légale. Si une telle confidentialisation du contentieux ne saurait ipso facto être analysée comme un alignement du rôle du Conseil d'Etat sur celui d'une autorité administrative, elle altère considérablement le droit des individus à un recours effectif et rend particulièrement opaque les possibilités de contestation de ces décisions puisque ce contentieux s'effectue en aveugle...

Ce sont des pas supplémentaires vers la fonction d'administrateur que la loi fait ensuite franchir au juge administratif -avec, notamment, les $4^{\text {ème }}$ et $5^{\text {ème }}$ lois de prorogation de l'état d'urgence - officiellement au nom de la lutte contre le terrorisme.

La loi du 21 juillet 2016 crée en effet un nouveau mode d'intervention du juge administratif : I'autorisation, en référé, de l'exploitation des données informatiques saisies lors d'une perquisition administrative ordonnée en application de la loi du 3 avril 1955. C'est la loi du 20 novembre 2015 qui avait autorisé l'autorité administrative à « copier toutes les données informatiques auxquelles il lui aura été possible d'accéder » au cours d'une perquisition (et donc, de procéder à une saisie sans toutefois qu'il n'y ait ni intervention d'un juge, ni même d'infraction constatée). Mais le Conseil constitutionnel, le 19 janvier 2016, jugeait que faisaient ici défaut les " garanties légales propres à assurer une conciliation équilibrée entre l'objectif de valeur constitutionnelle de sauvegarde de l'ordre public et le droit au respect de la vie privée ${ }^{56}$. C'est donc afin de chercher à corriger ce défaut d'inconstitutionnalité que le législateur entérinait quelques mois plus tard pat cette loi du 21 juillet 2016 le projet du gouvernement conférant au Conseil d'Etat, en référé, le pouvoir d'autoriser l'exploitation de données saisies ${ }^{57}$. Ainsi, dès lors qu'une perquisition donne lieu à la saisie d'appareils électroniques ou données informatiques, il appartient à l'autorité administrative de solliciter aussitôt une autorisation d'exploitation des données auprès du juge des référés du TA du ressort dans lequel a lieu la perquisition, lequel statue dans un délai de 48 heures. Si le juge refuse l'autorisation, les données copiées sont détruites et les supports restitués à leurs propriétaires ; s'il l'accorde, ils sont conservés pendant « le temps

\footnotetext{
55 Pour un commentaire du premier rapport, L. Chataignier, A. Geraud et T. Gauthier, « L'Etat de droit à l'épreuve du renseignement : Bilan du premier rapport d'activité de la CNCTR ", La Revue des droits de I'homme [En ligne], Actualités Droits-Libertés, 17 février 2017. URL : http://revdh.revues.org/3010

${ }^{56}$ CC, 2016-536QPC, $L D H$, préc.

${ }^{57} \mathrm{~V}$. sur l'opposition des syndicats de magistrats à ce référé a priori : " 3 questions à Hélène Bronnenkant ", AJDA, 2016 p.1540 et C. Tukov, « Le contrôle a priori confié au juge administratif pour l'exploitation par l'autorité administrative des données informatiques saisies dans le cadre d'une perquisition administrative a-til un sens ? ", JCP $A \mathrm{n}^{\circ}$ 35, 5 Sept. 2016, 2229 qui évoque un juge administratif des référés « en voie de JLDisation $"$.
} 
strictement nécessaire à leur exploitation » et en tout état de cause, pas au-delà de 15 jours (les données copiées devant, quant à elles, être détruites à l'expiration d'un délai de trois mois) -sauf prorogations, autorisées là encore par le juge administratif en cas de difficulté technique d'accès aux données, et sauf constatation d'une infraction pénale, auquel cas les données et supports changent de régime juridique et peuvent être conservés selon les règles applicables en matière de procédure pénale.

Comment analyser la mission ici confiée par le législateur au juge administratif ? Elle se rapproche assez fortement d'une mission administrative : il s'agit de délivrer des " autorisations d'exploitation » et ce " au vu des éléments révélés par la perquisition ", c'est à dire des éléments révélant « la menace que constitue pour la sécurité et l'ordre publics le comportement de la personne concernée ". Et de fait, l'observation des décisions rendues par les juridictions administratives sur ce fondement confirme que, sans disposer réellement des moyens de procéder à un contrôle approfondi des décisions de saisie, le juge se borne souvent à délivrer les autorisations d'exploitation dès lors qu'est remplie la condition de la présence d'images ou vidéos en lien avec le terrorisme sur les smartphones, tablettes ou ordinateurs saisis - étant entendu, en outre, que cette " présence » est parfois indirecte, comme c'est le cas lorsqu'est saisi téléphone contenant une application permettant d'accéder à une " vidéo montrant un drapeau de Daesh au milieu de combattants " et dans laquelle le nom d'un porte-parole de l'El était prononcé ${ }^{58}$. II faudra, là encore, encore un peu de temps pour pouvoir analyser de manière significative sinon exhaustive les décisions rendues par le juge administratif dans ce cadre ; mais des premiers éléments semblent corroborer l'idée que les refus d'autorisation sont très rares ${ }^{59}$, tout comme le sont les cas dans lesquels le juge exige davantage que des éléments attestant la présence de " vidéos djihadistes » sur les appareils saisis ${ }^{60}$.

Quant à la loi du 19 décembre 2016, elle poursuivait -avant d'être précisément censurée sur ce point ${ }^{61}$ - ce processus d'administrativisation de l'office du juge administratif, en créant elle aussi une nouvelle procédure d'autorisation délivrée par le juge en référé, en matière, cette fois, de prolongation des assignations à résidence "longue durée ". Si, en effet, la loi pose comme principe qu'une mesure d'assignation n'a pas vocation à durer plus de douze mois ${ }^{62}$, elle en organise aussitôt le double contournement. Au sujet des personnes déjà assignées depuis plus de douze mois à la date d'entrée en vigueur de la loi, elle prévoit d'abord un régime spécifique permettant, dans un délai de quatre-vingt-dix jours à compter de l'entrée en vigueur de loi, leur ré-assignation pour une durée maximale de quatre-vingtdix jours (art. 2.11 de la loi) ${ }^{63}$. La loi créait ensuite une nouvelle procédure devant le juge des référés du Conseil d'Etat, qui permettait la prolongation d'assignations ayant déjà produit leurs effets pendant un an : le juge devait alors statuer sur les raisons sérieuses invoquées par l'autorité administrative pour estimer que le « comportement de la personne continue à

\footnotetext{
${ }^{58}$ TA Paris, 3 sept. 2016, n¹613466/9.

${ }^{59}$ V. tout de même, par ex., TA Rennes, ord., 5 août 2016, n¹603447.

${ }^{60} \mathrm{~V}$. tout de même, par exemple, TA Pau, ord., 9 août 2016, $\mathrm{n}^{\circ} 1601533$, où le juge évoque de nombreux autres éléments que les indices informatiques saisis pour autoriser l'exploitation des appareils informatiques.

${ }^{61}$ CC, n² 2017-624 QPC, 16 mars 2017.

${ }^{62}$ Une telle durée est déjà considérable au regard du fait (faut-il le rappeler ?) qu'une telle mesure, qui constitue une restriction sévère de la liberté d'aller et venir et cause des difficultés importantes au regard de sa conciliation avec la vie privée et professionnelle, est préventive et peut trouver à s'appliquer à des personnes qui ne sont nullement mises en cause dans quelque procédure judiciaire que ce soit.

${ }^{63}$ Le ministère de l'Intérieur a pris cette possibilité au sérieux, signant plusieurs arrêtés de cette nature dès le 22 décembre 2016.
} 
constituer une menace pour la sécurité et l'ordre publics ». Rien n'excluant le cumul des deux dispositifs, il aurait été possible, si ce texte avait vécu, que le ministre demande au juge la prolongation pour quatre-vingt-dix jours de l'assignation d'une personne qui, assignée depuis un an à la date d'entrée en vigueur de la loi, aurait déjà fait l'objet d'une réassignation de quatre-vingt-dix jours sur le fondement de l'article 2.Il de la loi. On aurait alors vu apparaître des assignations de 12 mois +90 jours +90 jours $=18$ mois, les assignations " ultra longue durée ${ }^{64}$. L'horizon d'une telle évolution était d'autant plus inquiétante qu'on a constaté, depuis la dernière prolongation de l'état d'urgence, en décembre 2016, la multiplication des cas d'assignations à résidence, dès leur sortie de prison, d'individus en raison de leur " radicalisation » en détention ${ }^{65}$-configuration dans laquelle cette nouvelle forme déguisée de surveillance de sûreté vient concurrencer des formes de contrôle judiciaire. En toute hypothèse, ce dispositif pouvait être lu comme un avatar supplémentaire de la transformation récente de l'office même du juge administratif, tendanciellement appelé, sous la pression de l'état d'urgence dissimulé sous le discours de la nécessaire lutte contre le terrorisme, à se faire administrateur plus que juge. La question de la compatibilité entre ce nouveau pouvoir du juge administratif (autoriser la prolongation des assignations à résidence) et ses fonctions contentieuses classiques (qui peuvent l'amener à connaître de recours formés contre des prolongations d'assignations qu'il aurait lui-même autorisées) est d'ailleurs suffisamment sérieuse pour que le juge constitutionnel, saisi par voie de QPC, ait soulevé à ce propos un moyen d'ordre public... et, finalement, censuré le dispositif : " les dispositions contestées attribuent au Conseil d'État statuant au contentieux la compétence d'autoriser, par une décision définitive et se prononçant sur le fond, une mesure d'assignation à résidence sur la légalité de laquelle il pourrait ultérieurement avoir à se prononcer comme juge en dernier ressort. Dans ces conditions, ces dispositions méconnaissent le principe d'impartialité et le droit à exercer un recours juridictionnel effectif ${ }^{66}$. Les dispositions législatives prévoyant l'autorisation de prolongation des assignations sont donc déclarées inconstitutionnelles. Si la décision manifeste en ce sens la vigueur des principes d'impartialité de séparation des pouvoirs, elle demeure critiquable du point de vue de la protection des libertés à deux égards. D’abord, le Conseil constitutionnel ne trouve rien à redire sur le principe même des assignations longue durée (« la seule prolongation dans le temps d'une mesure d'assignation à résidence ordonnée dans les conditions prévues par l'article 6 de la loi du 3 avril 1955 n'a toutefois pas pour effet de modifier sa nature et de la rendre assimilable à une mesure privative de liberté. Dès lors, le grief tiré de la méconnaissance de l'article 66 de la Constitution doit être écarté »). Ensuite, la censure qu'il prononce, pour justifiée qu'elle apparaisse, laisse en vigueur un état du droit amputé : le ministre de l'Intérieur demeure seul décisionnaire ${ }^{67}$ pour ce qui est de ces prolongations des assignations longue durée...

\footnotetext{
${ }^{64}$ Ce dispositif a d'ailleurs rapidement suscité une question prioritaire de constitutionnalité transmise par le Conseil d'Etat (CE, ord., 16 janv. 2017, n406614) d'autant plus importante que les derniers bilans chiffrés de l'état d'urgence font état de plus de 90 personnes assignées au 14 décembre 2016, dont $40 \%$ (37 personnes) depuis plus d'un an..

${ }^{65}$ Ass. nat., Communication d'étape sur le contrôle de l'état d'urgence, par D. Raimbourg, JF Poisson, 22 février 2017.

${ }^{66}$ CC, 2017-624 QPC, 16 mars 2017.

${ }^{67}$ Le Conseil formule toutefois des réserves : l'autorisation ne peut être décidée que « sous réserve, d'une part, que le comportement de la personne en cause constitue une menace d'une particulière gravité pour la sécurité et l'ordre publics, d'autre part, que l'autorité administrative produise des éléments nouveaux ou complémentaires, et enfin que soient prises en compte dans l'examen de la situation de l'intéressé la durée
} 
Par-delà les transformations que cet attelage lutte contre le terrorisme / installation durable de l'état d'urgence imprime à la justice administrative stricto sensu, il faudrait encore compter avec les effets de diffusion d'une logique de l'exception au sein de l'ensemble de l'appareil administratif -un phénomène d'ampleur dont il serait probablement illusoire de penser que le juge administratif serait à même de le neutraliser, ou même de le cantonner. II n'est d'ailleurs pas anodin de ce point de vue que, dès les premiers mois de ce qui s'annonçait comme un enracinement durable de l'état d'urgence, un collectif anonyme de magistrats administratifs ait précisément entendu attirer l'attention sur ce danger ${ }^{68}$. En toute hypothèse, l'observation d'un an et demi de mise en pratique administrative et contentieuse de la loi du 3 avril 1955 révèle la multiplicité des usages des pouvoirs spéciaux permis par ce texte dans des situation dépourvues de tout lien avec la problématique terroriste, mais que l'autorité administrative qualifie de menace sécuritaire. Le droit des étrangers comme les moyens déployés face à la " crise des migrants " constituent ici des illustrations. D'autres usages sont, plus clairement encore, de simples outils de gestion et de maintien de l'ordre ; le sort réservé, depuis novembre 2015 , à la liberté de manifestation est exemplaire de ce point de vue. L'ensemble de ces mesures administratives, prises au cœur ou en marge de l'état d'urgence et tantôt légitimées, tantôt invisibilisées par la lutte contre le terrorisme, est une marque durable dans le fonctionnement de l'appareil d'Etat, mais aussi, pour la part qui en aura connu des développements contentieux, de l'appareil juridictionnel. Nous avons livré ici quelques premières pistes d'analyse, qui ont vocation à être complétées. II faut analyse le droit administratif de l'urgence.

\footnotetext{
totale de son placement sous assignation à résidence, les conditions de celle-ci et les obligations complémentaires dont cette mesure a été assortie ", étant entendu que la durée de l'assignation doit être justifiée et proportionnée aux raisons ayant motivé la mesure dans les circonstances particulières ayant conduit à la déclaration de l'état d'urgence -caractéristiques qu'il appartient au juge administratif de contrôler : CC, 2017-624 QPC, 16 mars 2017, §17-18.

68 https://blogs.mediapart.fr/edition/les-invites-de-mediapart/article/291215/etat-d-urgence-des-jugesadministratifs-appellent-la-prudence
} 